\title{
MODELAGEM MATEMÁTICA E DIFUSIVIDADE EFETIVA DAS SEMENTES DE PINHÃO-MANSO (Jatropha curcas L.) DURANTE A SECAGEM
}

\section{OSVALDO RESENDE ${ }^{1}$, RENAN ULLMANN ${ }^{2}$, VALDINEY C. SIQUEIRA ${ }^{3}$, TARCÍSIO H. CHAVES ${ }^{4}$, LÊNIO U. FERREIRA ${ }^{5}$}

\begin{abstract}
RESUMO: O presente trabalho teve o objetivo de ajustar diferentes modelos matemáticos aos dados experimentais da secagem de sementes de pinhão-manso (Jatropha curcas L.), bem como determinar o coeficiente de difusão efetivo e obter a energia de ativação para a faixa de temperatura utilizada. As sementes de pinhão-manso com teor de água de 0,67 (decimal b.s.) passaram por um período de pré-secagem em ambiente natural para reduzir e homogeneizar o teor de água para 0,30 (decimal b.s.). Em seguida, a secagem foi realizada em secador experimental mantendo-se as temperaturas controladas de $30 ; 40 ; 50 ; 60$ e $70 \pm 1{ }^{\circ} \mathrm{C}$ e umidades relativas de 55,$98 ; 41,44 ; 35,35$; 26,21 e 13,37 $\pm 3 \%$, respectivamente. Aos dados experimentais foram ajustados onze modelos matemáticos utilizados para a representação do processo de secagem de produtos agrícolas. Conclui-se que, dentre os modelos analisados, Page e Henderson e Pabis Modificado apresentaram os melhores ajustes aos dados experimentais, sendo o modelo de Page selecionado para a descrição das curvas de secagem do pinhão-manso devido a sua simplicidade; o coeficiente de difusão efetiva aumenta com a elevação da temperatura, apresentando magnitudes entre $3,93 \times 10^{-10} \mathrm{e}$ $9,19 \times 10^{-10} \mathrm{~m}^{2} \mathrm{~s}^{-1}$ para o intervalo de temperatura de 30 a $70{ }^{\circ} \mathrm{C}$, respectivamente; e a energia de ativação para a difusão líquida do pinhão-manso durante a secagem foi de $15,781 \mathrm{~kJ} \mathrm{~mol}^{-1}$.
\end{abstract}

PALAVRAS-CHAVE: curvas de secagem, modelos matemáticos, energia de ativação.

\section{MATHEMATICAL MODELING AND EFFECTIVE DIFFUSION OF JATROPHA (Jatropha curcas L.) SEEDS DURING DRYING}

\begin{abstract}
The aim of this study was to adjust different mathematical models to experimental data for jatropha seed drying (Jatropha curcas L.) and determine the effective diffusion coefficient and obtain the activation energy for the temperature range used. Jatropha seeds harvested with moisture content of 0.67 (decimal d.b.) and dried in a natural environment to reduce and homogenize the moisture content for 0.30 (decimal d.b.). Jatropha drying was performed in experimental drier maintained at controlled temperatures of $30 ; 40 ; 50 ; 60$ or $70 \pm 1{ }^{\circ} \mathrm{C}$ and relative humidity of $55.98 ; 41.44 ; 35.35 ; 26.21$ and $13.37 \pm 3 \%$, respectively. Experimental data were fitted to eleven mathematical models used to represent the process of drying of agricultural products. It was concluded that among the models tested, Page and Henderson and Modified Pabis presented the best fitting to experimental data, and the Page model selected for describing the drying curves from jatropha because of their simplicity; the effective diffusion coefficient increases with increasing temperature, with magnitudes between $3.93 \times 10^{-10}$ and $9.19 \times 10^{-10} \mathrm{~m}^{2} \mathrm{~s}^{-1}$ for the temperature range $30-70{ }^{\circ} \mathrm{C}$, respectively. The activation energy for liquid diffusion during jatropha drying was $15.781 \mathrm{~kJ} \mathrm{~mol}^{-1}$.
\end{abstract}

KEYWORDS: drying curves, mathematical models, activation energy.

\footnotetext{
${ }^{1}$ Eng ${ }^{\underline{0}}$ Agrícola, Prof. Dr., IF Goiano, Câmpus Rio Verde, Rodovia Sul Goiana, km 01, Zona Rural, Rio Verde, GO, osvresende@yahoo.com.br.

${ }^{2}$ Graduando em Agronomia, IF Goiano, Câmpus Rio Verde, Rodovia Sul Goiana, km 01, Zona Rural, Rio Verde, GO, renanullmann@hotmail.com - Bolsista PIBIC/CNPq.

${ }^{3}$ Agrônomo, Doutorando em Engenharia Agrícola, UFLA, vcambuy@ yahoo.com.

${ }^{4}$ Graduando em Agronomia, IF Goiano, Câmpus Rio Verde, Rodovia Sul Goiana, km 01, Zona Rural, Rio Verde, GO, tarcisio.chaves@hotmail.com - Bolsista PIBIC/CNPq.

${ }^{5}$ Graduando em Agronomia, IF Goiano, Câmpus Rio Verde, Rodovia Sul Goiana, km 01, Zona Rural, Rio Verde, GO, lenio_urzeda@hotmail.com.

Recebido pelo Conselho Editorial em: 9-8-2010
}

Aprovado pelo Conselho Editorial em: 15-8-2011 


\section{INTRODUÇÃO}

Com o incentivo criado pelo Governo Federal brasileiro a partir do Programa de Biodiesel, o plantio de áreas com pinhão-manso (Jatropha curcas L.) vem crescendo, tanto por pequenos agricultores como por empresas agrícolas que buscam explorar novos nichos de mercado; isso se deve principalmente às várias vantagens que o pinhão-manso apresenta em relação à mamona (oleaginosa indicada pelo governo como primeira escolha para projetos relacionados à agricultura familiar), entre elas: menor exigência hídrica e nutricional, capacidade de recuperação de áreas degradadas em função de suas raízes profundas, além de apresentar maior produtividade média (TEIXEIRA, 2005). Logo, a cultura do pinhão-manso tem o potencial de se adaptar às condições adversas ao seu desenvolvimento, podendo ser cultivada praticamente em todas as regiões do Brasil.

Na fase de pós-colheita dos produtos vegetais, a secagem é o processo mais utilizado para assegurar sua qualidade e estabilidade, considerando que a redução do teor de água do material reduz a atividade biológica e as mudanças químicas e físicas que ocorrem durante $\mathrm{o}$ armazenamento. Este fenômeno de redução do teor de água dos grãos envolve um processo simultâneo de transferência de calor e massa, que, normalmente, ocorre entre o ar aquecido e a massa de água presente nos grãos por meio de convecção. Assim, é de fundamental importância o melhor entendimento deste processo para se ter uma secagem eficiente, do ponto de vista técnico e econômico.

No desenvolvimento e aperfeiçoamento de equipamentos utilizados para a secagem de grãos, são de fundamental importância a simulação e a obtenção de informações teóricas a respeito do comportamento de cada produto durante a remoção de água. Para a simulação, cujo princípio se fundamenta na secagem de sucessivas camadas delgadas do produto, utiliza-se um modelo matemático que representa, satisfatoriamente, sua perda de água durante o período de secagem (BERBERT et al., 1995; GINER \& MASCHERONI, 2002).

As curvas de secagem, em camada delgada, variam com a espécie, variedade, condições ambientais, métodos de preparo pós-colheita, entre outros fatores. Neste sentido, diversos modelos matemáticos têm sido utilizados para descrever o processo de secagem de produtos agrícolas (RESENDE et al., 2008). Esses modelos, geralmente, baseiam-se em variáveis externas ao produto, como a temperatura e a umidade relativa do ar de secagem. Entretanto, não fornecem indicações detalhadas sobre os fenômenos de transporte de energia e de água no interior dos grãos e consideram que todo o processo de secagem ocorre somente no período de taxa decrescente.

Segundo MIDILLI et al. (2002), existem três tipos de modelos de secagem em camada delgada que visam a descrever a cinética de secagem de produtos agrícolas. O modelo teórico, que considera apenas a resistência interna, a transferência de calor e a água entre o produto e o ar quente; os modelos semiteóricos e os empíricos, que consideram somente a resistência interna, a temperatura e a umidade relativa do ar de secagem.

A difusão de água em produtos agrícolas durante a secagem é um processo complexo que pode envolver diferentes mecanismos, como a difusão molecular, difusão capilar, difusão de superfície, fluxo hidrodinâmico, difusão de vapor e difusão térmica (GONELI et al., 2009). A teoria da difusão líquida (modelo teórico) assume que não há influência da capilaridade, despreza os efeitos da transferência de energia e massa de um corpo para outro, e também considera que os corpos entram em equilíbrio térmico com o ar, instantaneamente. Pode-se dizer que o coeficiente de difusão descreve a velocidade de saída de água do grão. Como a difusividade varia conforme mudam as condições de secagem, ela não é intrínseca ao material, e assim, convenciona-se chamála de difusividade efetiva (OLIVEIRA et al., 2006).

Diante do destaque da cultura do pinhão-manso no cenário nacional e internacional e da falta de informações teóricas a respeito do comportamento das sementes dessa cultura durante o processo de secagem, objetivou-se com o presente trabalho ajustar diferentes modelos matemáticos aos dados 
experimentais da secagem de sementes de pinhão-manso e selecionar aquele que melhor representa o fenômeno, além de determinar o coeficiente de difusão efetivo e obter a energia de ativação para o processo de secagem do pinhão-manso submetido a cinco condições de ar.

\section{MATERIAL E MÉTODOS}

O presente trabalho foi realizado no Laboratório de Pós-Colheita de Produtos Vegetais e no Laboratório de Sementes do Instituto Federal de Educação, Ciência e Tecnologia Goiano - Câmpus Rio Verde (IF Goiano - Câmpus Rio Verde).

A colheita dos frutos e o beneficiamento das sementes foram realizados manualmente, tendo as sementes o teor de água inicial de, aproximadamente, 0,67 (decimal b.s.). Em seguida, as sementes ficaram expostas ao ar natural para reduzir e homogeneizar seu teor de água até 0,30 (decimal b.s.). A secagem foi realizada em estufa com ventilação de ar forçada, com temperaturas controladas de 30; 40; 50; 60 e $70 \pm 1{ }^{\circ} \mathrm{C}$ e umidades relativas de 56,0; 41,4; 35,3; 26,2 e 13,4 $\pm 3 \%$, respectivamente, em camada fina.

Durante o processo de secagem, as bandejas de aço com fundo perfurado, contendo 275 gramas de produto, totalizando uma altura de $12 \pm 2 \mathrm{~cm}$ na bandeja, foram pesadas, juntamente com o produto, periodicamente, até atingirem teores de água de 0,09 $\pm 0,01$ (decimal b.s.) definido como o teor de água recomendado para armazenamento seguro das sementes. As amostras foram pesadas em balanças analíticas da marca "Shimadzu", com resolução de $0,01 \mathrm{~g}$.

Os teores de água das sementes foram determinados por gravimetria, utilizando-se da estufa a $105 \pm 1{ }^{\circ} \mathrm{C}$, durante 24 horas, em três repetições (BRASIL, 2009).

A temperatura e a umidade relativa do ar de secagem foram monitoradas por meio de um psicrômetro instalado no interior da estufa. Para a determinação das razões de umidade do pinhão-manso durante a secagem, utilizou-se a seguinte expressão:

$$
\mathrm{RX}=\frac{\mathrm{X}^{*}-\mathrm{X}_{\mathrm{e}}^{*}}{\mathrm{X}_{\mathrm{i}}^{*}-\mathrm{X}_{\mathrm{e}}^{*}}
$$

em que,

$\mathrm{RX}$ - razão de teor de água, adimensional;

$\mathrm{X}^{*}$ - teor de água do produto (decimal b.s.);

$\mathrm{X}_{\mathrm{i}}^{*}$ - teor de água inicial do produto (decimal b.s.), e

$\mathrm{X}_{\mathrm{e}}^{*}$ - teor de água de equilíbrio do produto (decimal b.s.).

O teor de água de equilíbrio dos grãos de pinhão-manso em cada temperatura foi obtido experimentalmente. As bandejas contendo as amostras permaneceram na estufa até que a massa do produto permanecesse invariável durante três pesagens consecutivas.

Aos dados experimentais da secagem do pinhão-manso ajustaram-se os modelos matemáticos frequentemente utilizados para representação da secagem de produtos agrícolas, conforme apresentação na Tabela 1. 
TABELA 1. Modelos matemáticos utilizados para predizer a secagem de produtos agrícolas. Mathematical models used to predict the drying of agricultural products.

\begin{tabular}{lr}
\hline Designação do modelo & Modelo \\
$\mathrm{RX}=1+\mathrm{at}+\mathrm{b} \mathrm{t}^{2}$ & Wang e Sing (WANG \& SING, 1978) \\
$\mathrm{RX}=\mathrm{a} \cdot \exp (-\mathrm{k} \cdot \mathrm{t})+(1-\mathrm{a}) \exp \left(-\mathrm{k}_{1} \cdot \mathrm{t}\right)$ & Verma (VERMA et al., 1985) \\
$\mathrm{RX}=\exp \left(\left(-\mathrm{a}-\left(\mathrm{a}^{2}+\mathrm{t} \cdot \mathrm{b} \cdot \mathrm{t}\right)^{0,5}\right) / 2 \cdot \mathrm{b}\right)$ & Thompson (THOMPSON et al., 1968) \\
$\mathrm{RX}=\exp \left(-\mathrm{k} \cdot \mathrm{t}^{\mathrm{n}}\right)$ & Page (PAGE, 1949) \\
$\mathrm{RX}=\exp (-\mathrm{k} \cdot \mathrm{t})$ & Midilli (MIDILLI, 2002) \\
$\mathrm{RX}=\mathrm{a} \cdot \exp \left(-\mathrm{k} \cdot \mathrm{t}^{\mathrm{n}}\right)+\mathrm{b} \cdot \mathrm{t}$ & Logarítmico (YAGCIOGLU et al., 1999) \\
$\mathrm{RX}=\mathrm{a} \cdot \exp (-\mathrm{k} \cdot \mathrm{t})+\mathrm{c}$ & Henderson e Pabis \\
$\mathrm{RX}=\mathrm{a} \cdot \exp (-\mathrm{k} \cdot \mathrm{t})$ & (HENDERSON \& PABIS, 1961) \\
$\mathrm{RX}=\mathrm{a} \cdot \exp (-\mathrm{k} \cdot \mathrm{t})+\mathrm{b} \cdot \exp \left(-\mathrm{k}_{\mathrm{o}} \cdot \mathrm{t}\right)+\mathrm{c} \cdot \exp \left(-\mathrm{k}_{1} \cdot \mathrm{t}\right)$ & Henderson e Pabis modificado \\
$\mathrm{RX}=\mathrm{a} \cdot \exp (-\mathrm{k} \cdot \mathrm{t})+(1-\mathrm{a}) \exp (-\mathrm{k} \cdot \mathrm{a} \cdot \mathrm{t})$ & Exponencial de Dois Termos \\
$\mathrm{RX}=\mathrm{a} \cdot \exp (-\mathrm{k} \mathrm{o} \cdot \mathrm{t})+\mathrm{b} \cdot \exp \left(-\mathrm{k}_{1} \cdot \mathrm{t}\right)$ & (SHARAF-ELDEE et al., 1980) \\
\hline
\end{tabular}

em que,

$\mathrm{t}$ - tempo de secagem, $\mathrm{h}$;

$\mathrm{k}, \mathrm{k}_{\mathrm{o}}, \mathrm{k}_{1}$ - constantes de secagem, e

$\mathrm{a}, \mathrm{b}, \mathrm{c}, \mathrm{n}$ - coeficientes dos modelos.

Para o ajuste dos modelos matemáticos, realizou-se análise de regressão não linear, pelo método Gauss Newton, utilizando o programa STATISTICA 7.0 ${ }^{\circledR}$. Para verificar o grau de ajuste de cada modelo, foi considerada a magnitude do coeficiente de determinação $\left(\mathrm{R}^{2}\right)$, do erro médio relativo $(\mathrm{P})$ e do erro médio estimado (SE) e a distribuição de resíduos. O erro médio relativo e o erro médio estimado, para cada um dos modelos, foram calculados conforme as seguintes expressões:

$$
\begin{aligned}
& \mathrm{P}=\frac{100}{\mathrm{n}} \sum \frac{|\mathrm{Y}-\hat{\mathrm{Y}}|}{\mathrm{Y}} \\
& \mathrm{SE}=\sqrt{\frac{\sum(\mathrm{Y}-\hat{\mathrm{Y}})^{2}}{\mathrm{GLR}}}
\end{aligned}
$$

em que,

Y - valor observado experimentalmente (razão de teor de água);

$\hat{Y}$ - valor estimado pelo modelo (razão de teor de água), e

$\mathrm{n}$ - número de observações experimentais.

GLR - grau de liberdade do modelo (número de observações menos o número de parâmetros do modelo). 
A solução analítica do modelo da difusão líquida para a forma geométrica cilíndrica, com aproximação de oito termos (eq.(15)), foi ajustada aos dados experimentais de secagem do pinhãomanso considerando o raio equivalente inicial dos grãos e mantendo-o constante ao longo da secagem.

$$
\mathrm{RX}=\frac{\mathrm{X}^{*}-\mathrm{X}_{\mathrm{e}}^{*}}{\mathrm{X}_{\mathrm{i}}^{*}-\mathrm{X}_{\mathrm{e}}^{*}}=\sum_{\mathrm{n}=1}^{\infty} \frac{4}{\lambda_{\mathrm{n}}^{2}} \exp \left[-\frac{\lambda_{\mathrm{n}}^{2} \cdot \mathrm{D} \cdot \mathrm{t}}{\mathrm{r}}\right]
$$

em que,

$\mathrm{D}$ - coeficiente de difusão efetivo, $\mathrm{m}^{2} \mathrm{~s}^{-1}$;

n - número de termos;

$\lambda_{\mathrm{n}}$ - raízes da equação de Bessel de ordem zero;

$\mathrm{r}$ - raio de esfera equivalente, $0,01346 \mathrm{~m}$, e

$\mathrm{t}$ - tempo de secagem, $\mathrm{s}$.

Adotaram-se as seguintes condições de contorno para a obtenção da solução analítica (BROOKER et al., 1992):

$$
\begin{aligned}
& X^{*}(r, 0)=X_{i}^{*} \\
& X^{*}(r, t)=X_{e}^{*}
\end{aligned}
$$

O raio equivalente é definido como o raio de uma esfera com volume equivalente ao do grão. O volume ( $\mathrm{Vg}$ ) de cada grão foi obtido por meio da medição dos três eixos ortogonais (comprimento, largura e espessura), em vinte grãos, no início da secagem, com auxílio de um paquímetro digital, de acordo com a expressão proposta por MOHSENIN (1986):

$$
\mathrm{Vg}=\frac{\pi \cdot(\mathrm{a} \cdot \mathrm{b} \cdot \mathrm{c})}{6}
$$

em que,

$$
\begin{aligned}
& \text { a - maior eixo do grão, m; } \\
& \text { b - eixo médio do grão, m, e } \\
& \text { c - menor eixo do grão, } m \text {. }
\end{aligned}
$$

A relação do aumento do coeficiente de difusão, com a elevação da temperatura do ar de secagem, foi descrita por meio da equação de Arrhenius.

$$
\mathrm{D}=\mathrm{D}_{\mathrm{o}} \cdot \exp \left(\frac{-\mathrm{E}_{\mathrm{a}}}{\mathrm{R} \cdot \mathrm{T}_{\mathrm{a}}}\right)
$$

em que,

$\mathrm{D}_{\mathrm{o}}$ - fator pré-exponencial;

$\mathrm{E}_{\mathrm{a}}$ - energia de ativação, $\mathrm{kJ} \mathrm{mol}^{-1}$;

$\mathrm{R}$ - constante universal dos gases, $8,134 \mathrm{~kJ} \mathrm{kmol}^{-1} \mathrm{~K}^{-1}$, e

$\mathrm{T}_{\mathrm{a}}$ - temperatura absoluta, $\mathrm{K}$.

Os coeficientes da expressão de Arrhenius podem ser facilmente obtidos linearizando a eq.(19) com a aplicação do logaritmo na seguinte forma:

$$
\mathrm{LnD}=\mathrm{LnD}_{\mathrm{o}}-\frac{\mathrm{E}_{\mathrm{a}}}{\mathrm{R}} \cdot \frac{1}{\mathrm{~T}_{\mathrm{a}}}
$$




\section{RESULTADOS E DISCUSSÃO}

Na Tabela 2, estão apresentados os valores do erro médio relativo estimado (SE), erro médio relativo $(\mathrm{P})$ e do coeficiente de determinação $\left(\mathrm{R}^{2}\right)$ para os modelos ajustados à cinética de secagem do pinhão-manso em diferentes temperaturas.

TABELA 2. Coeficientes de determinação $\left(\mathrm{R}^{2}, \%\right)$, erros médio relativo $(\mathrm{P}, \%)$ e estimado (SE, decimal) para os onze modelos analisados, durante a secagem do pinhão-manso, nas diversas condições de temperatura $\left({ }^{\circ} \mathrm{C}\right)$. Coefficients of determination (R2, \%), average relative errors $(P \%)$ and estimated (SE, decimal) for the eleven models analyzed during the jatropha drying in several temperature conditions $\left({ }^{\circ} \mathrm{C}\right)$.

\begin{tabular}{|c|c|c|c|c|c|c|c|c|c|c|c|c|c|c|c|}
\hline \multirow[b]{2}{*}{ Modelo } & \multicolumn{3}{|c|}{$30^{\circ} \mathrm{C}$} & \multicolumn{3}{|c|}{$40^{\circ} \mathrm{C}$} & \multicolumn{3}{|c|}{$50{ }^{\circ} \mathrm{C}$} & \multicolumn{3}{|c|}{$60{ }^{\circ} \mathrm{C}$} & \multicolumn{3}{|c|}{$70^{\circ} \mathrm{C}$} \\
\hline & SE & $\mathrm{P}$ & $\mathrm{R}^{2}$ & SE & $\mathrm{P}$ & $\mathrm{R}^{2}$ & SE & $\mathrm{P}$ & $\mathrm{R}^{2}$ & SE & $\mathrm{P}$ & $\mathrm{R}^{2}$ & SE & $\mathrm{P}$ & $\mathrm{R}^{2}$ \\
\hline 2 & 0,015 & 3,36 & 99,88 & 0,019 & 3,47 & 99,68 & 0,011 & 1,67 & 99,88 & 0,006 & 0,71 & 99,97 & 0,017 & 1,65 & 99,78 \\
\hline 3 & 0,009 & 2,06 & 99,95 & 0,006 & 0,82 & 99,98 & 0,007 & 1,06 & 99,96 & 0,009 & 0,99 & 99,95 & 0,039 & 4,63 & 99,05 \\
\hline 4 & 0,023 & 7,75 & 99,71 & 0,007 & 1,28 & 99,96 & 0,007 & 1,09 & 99,95 & 0,008 & 0,98 & 99,95 & 0,020 & 2,94 & 99,68 \\
\hline 5 & 0,015 & 4,61 & 99,88 & 0,006 & 0,90 & 99,97 & 0,006 & 1,05 & 99,96 & 0,008 & 1,00 & 99,95 & 0,021 & 3,15 & 99,66 \\
\hline 6 & 0,022 & 7,74 & 99,71 & 0,017 & 2,95 & 99,74 & 0,007 & 1,05 & 99,95 & 0,007 & 0,98 & 99,95 & 0,032 & 4,63 & 99,06 \\
\hline 7 & 0,061 & 15,61 & 98,25 & 0,007 & 0,89 & 99,97 & 0,025 & 3,13 & 99,54 & 0,007 & 0,75 & 99,98 & 0,023 & 2,58 & 99,75 \\
\hline 8 & 0,009 & 1,98 & 99,96 & 0,009 & 1,53 & 99,94 & 0,007 & 0,97 & 99,96 & 0,017 & 2,27 & 99,79 & 0,021 & 2,74 & 99,73 \\
\hline 9 & 0,022 & 7,20 & 99,75 & 0,014 & 2,10 & 99,84 & 0,006 & 1,02 & 99,96 & 0,007 & 1,00 & 99,95 & 0,032 & 4,20 & 99,23 \\
\hline 10 & 0,010 & 1,98 & 99,96 & 0,006 & 0,83 & 99,98 & 0,009 & 1,02 & 99,96 & 0,013 & 1,01 & 99,95 & 0,045 & 2,91 & 99,69 \\
\hline 11 & 0,023 & 7,74 & 99,71 & 0,007 & 1,30 & 99,96 & 0,007 & 1,05 & 99,95 & 0,008 & 0,98 & 99,95 & 0,035 & 4,60 & 99,06 \\
\hline 12 & 0,023 & 7,20 & 99,75 & 0,006 & 0,83 & 99,98 & 0,007 & 1,02 & 99,96 & 0,009 & 1,01 & 99,95 & 0,041 & 4,20 & 99,23 \\
\hline
\end{tabular}

Verifica-se que, em relação aos coeficientes de determinação $\left(\mathrm{R}^{2}\right)$ referentes aos modelos matemáticos ajustados, nota-se que, com exceção do modelo de Midilli para a temperatura de $30{ }^{\circ} \mathrm{C}$, os demais valores apresentaram-se superiores a 99\%, que, de acordo com MADAMBA et al. (1996), indicam uma representação satisfatória do fenômeno em estudo. No entanto, o mesmo autor ressalta que apenas o coeficiente de determinação não constitui um bom critério para a seleção de modelos não lineares; logo, tornam-se necessárias avaliações de outros parâmetros, como o erro médio estimado e o erro médio relativo e a distribuição dos valores residuais.

Observa-se que, para os modelos analisados, o erro médio estimado (SE), que descreve o valor do desvio-padrão da estimativa, apresenta-se com uma alta variação, entre os modelos e as respectivas temperaturas em estudo. Contudo, os modelos de Verma (3), Thompson (4), Page (5), Midilli (7), Henderson e Pabis Modificado (10), Exponencial de Dois Termos (11) e Dois Termos apresentaram os menores valores do SE para a maioria das condições de secagem. Vale salientar que quanto menor for a valor do SE, melhor será a qualidade do ajuste do modelo em relação aos dados experimentais. Ainda na Tabela 2, observa-se que todos os modelos, exceto o de Midilli (7), apresentaram valores do erro médio relativo inferiores a 10\%, que, de acordo com MOHAPATRA \& RAO (2005), indica uma representação adequada do fenômeno.

$\mathrm{Na}$ Tabela 3, são descritos os comportamentos (ou tendências) da distribuição de resíduos para os modelos estudados. Verifica-se que os modelos de Page (5) e Henderson e Pabis Modificado (10) apresentaram uma distribuição aleatória dos resíduos, exceto para a temperatura de secagem de $50{ }^{\circ} \mathrm{C}$, na qual as distribuições dos resíduos se apresentaram de forma tendenciosa para todos os modelos. Um modelo é considerado aleatório se os valores residuais se encontrarem próximos à faixa horizontal em torno de zero e também não formarem figuras definidas, não indicando tendenciosidade dos resultados. Se apresentar distribuição tendenciosa, o modelo é considerado inadequado para representar o fenômeno em questão (GONELI et al., 2011). 
TABELA 3. Distribuição dos resíduos $(\mathrm{A}=$ aleatória; $\mathrm{T}=$ tendenciosa $)$ para os onze modelos analisados, durante a secagem do pinhão-manso nas diversas condições de temperatura. Residues Distribution $(\mathbf{A}=\operatorname{random}, \mathbf{T}=$ biased $)$ for the eleven models analyzed during the jatropha drying in several temperature conditions.

\begin{tabular}{cccccc}
\hline \multirow{2}{*}{ Modelos } & \multicolumn{5}{c}{ Temperatura $\left({ }^{\circ} \mathrm{C}\right)$} \\
\cline { 2 - 6 } & 30 & 40 & 50 & 60 & 70 \\
\hline 2 & $\mathrm{~A}$ & $\mathrm{~T}$ & $\mathrm{~T}$ & $\mathrm{~A}$ & $\mathrm{~A}$ \\
3 & $\mathrm{~A}$ & $\mathrm{~A}$ & $\mathrm{~T}$ & $\mathrm{~A}$ & $\mathrm{~T}$ \\
4 & $\mathrm{~T}$ & $\mathrm{~A}$ & $\mathrm{~T}$ & $\mathrm{~A}$ & $\mathrm{~A}$ \\
5 & $\mathrm{~A}$ & $\mathrm{~A}$ & $\mathrm{~T}$ & $\mathrm{~A}$ & $\mathrm{~A}$ \\
6 & $\mathrm{~T}$ & $\mathrm{~T}$ & $\mathrm{~T}$ & $\mathrm{~A}$ & $\mathrm{~T}$ \\
7 & $\mathrm{~T}$ & $\mathrm{~A}$ & $\mathrm{~T}$ & $\mathrm{~A}$ & $\mathrm{~A}$ \\
8 & $\mathrm{~A}$ & $\mathrm{~T}$ & $\mathrm{~T}$ & $\mathrm{~T}$ & $\mathrm{~A}$ \\
9 & $\mathrm{~T}$ & $\mathrm{~T}$ & $\mathrm{~T}$ & $\mathrm{~A}$ & $\mathrm{~T}$ \\
10 & $\mathrm{~A}$ & $\mathrm{~A}$ & $\mathrm{~T}$ & $\mathrm{~A}$ & $\mathrm{~A}$ \\
11 & $\mathrm{~T}$ & $\mathrm{~A}$ & $\mathrm{~T}$ & $\mathrm{~A}$ & $\mathrm{~T}$ \\
12 & $\mathrm{~T}$ & $\mathrm{~A}$ & $\mathrm{~T}$ & $\mathrm{~A}$ & $\mathrm{~T}$ \\
\hline
\end{tabular}

Devido ao menor número de coeficientes e, consequentemente, menor complexidade, o modelo de Page (5) foi escolhido para a representação do fenômeno da secagem das sementes de pinhão-manso. Diversos pesquisadores observaram que o tradicional modelo de Page foi recomendado e aplicado para predizer o fenômeno de secagem de vários produtos agrícolas: feijão-vermelho (CORRÊA et al., 2007), sementes de feijão-preto (AFONSO JÚNIOR \& CORRÊA, 1999), tomate (DOYMAZ, 2007), sementes de amaranto (ABALONE et al., 2006), polpa de maçã (WANG et al., 2007), café arábica (GONELI et al., 2009), mamona (GONELI, 2008), tarragon (ARABHOSSEINI et al., 2009), café (RESENDE et al., 2009a), dentre outros.

Na Tabela 4, estão apresentados os coeficientes do modelo de Page ajustados aos dados experimentais da cinética de secagem do pinhão-manso em diferentes temperaturas.

TABELA 4. Parâmetros dos modelos de Page ajustados para as diferentes condições de secagem das sementes de pinhão-manso. Parameters of Page model adjusted for different conditions of Jatropha seed drying.

\begin{tabular}{cccccc}
\hline \multirow{2}{*}{ Coeficientes } & \multicolumn{5}{c}{ Temperatura $\left({ }^{\circ} \mathrm{C}\right)$} \\
\cline { 2 - 5 } & 30 & 40 & 50 & 60 & 70 \\
\hline $\mathrm{k}$ & 0,1317 & 0,2430 & 0,2329 & 0,2899 & 0,4368 \\
$\mathrm{n}$ & 1,0983 & 0,9013 & 0,9768 & 1,0159 & 0,8263 \\
\hline
\end{tabular}

Verifica-se, na Tabela 4, que a magnitude da constante de secagem (k), que, de acordo com GONELI et al. (2009), representa o efeito das condições externas de secagem, tende a aumentar com a elevação da temperatura do ar de secagem, embora tenha sido sensivelmente menor na temperatura de $50{ }^{\circ} \mathrm{C}$ em relação à de $40{ }^{\circ} \mathrm{C}$. Segundo MADAMBA et al. (1996) e BABALIS \& BELESSIOTIS (2004), a constante de secagem (k) pode ser utilizada como uma aproximação para caracterizar o efeito da temperatura e está relacionada com a difusividade efetiva no processo de secagem para o período decrescente, sendo a difusão líquida que controla o processo. Vale salientar que, no processo de secagem durante qualquer fase de escoamento da água, os fatores externos, como a temperatura, a umidade relativa e a pressão de vapor são os que mais influenciam, uma vez que a saída de água do produto ocorre em função da diferença entre as características do ar de secagem e do produto submetido ao processo.

Com relação ao coeficiente (n) do modelo de Page, que de acordo com MISRA \& BROOKER, (1980), citado por ANDRADE et al. (2006), reflete a resistência interna do produto à 
secagem, não se observou uma tendência definida dos seus valores em relação à temperatura de secagem. Resultados semelhantes foram encontrados por GONELI et al. (2009), para a cinética de secagem dos grãos de café descascados em camada delgada. Normalmente, com a elevação da temperatura do ar de secagem menor o valor de "n", uma vez que há maior diferença entre a pressão de vapor do ar e do grão, promovendo maior remoção de água.

Na Figura 1, estão apresentadas as curvas de secagem das sementes de pinhão-manso estimadas pelo modelo de Page (5). Verifica-se o adequado ajuste do modelo pela correspondência entre os valores experimentais e estimados.

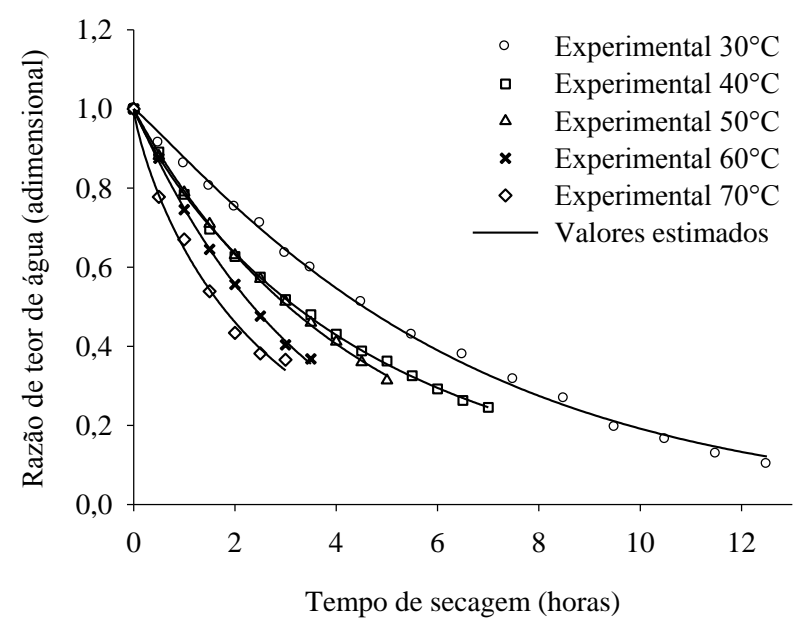

FIGURA 1. Valores experimentais da razão de umidade e estimados pelo modelo de Page para a secagem do pinhão-manso nas diversas condições de temperatura. Experimental values of the moisture and estimated ratio by Page model for jatropha drying in several temperature conditions.

Na Figura 2, são apresentados os valores médios do coeficiente de difusão efetivo para sementes de pinhão-manso submetidas a diferentes temperaturas do ar de secagem.

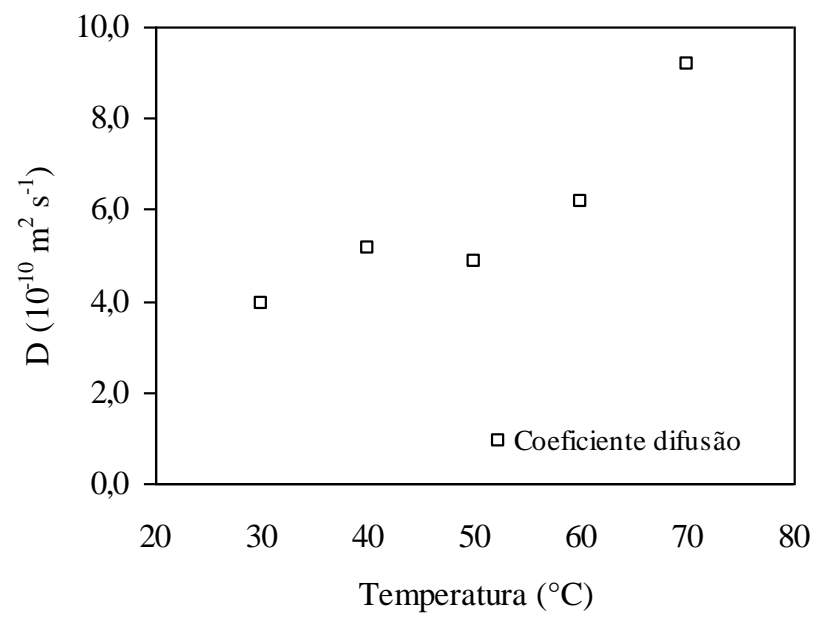

FIGURA 2. Valores médios do coeficiente de difusão efetivo $\left(10^{-10} \mathrm{~m}^{2} \mathrm{~s}^{-1}\right)$ obtidos para a secagem do pinhão-manso nas temperaturas de 30;40;50; 60 e $70{ }^{\circ} \mathrm{C}$. Mean values for the effective diffusion coefficient $\left(10^{-10} \mathrm{~m}^{2} \mathrm{~s}^{-1}\right)$ obtained for the jatropha drying at temperatures of $30 ; 40 ; 50 ; 60$ and $70{ }^{\circ} \mathrm{C}$.

Verifica-se, na Figura 2, que, durante a secagem, os coeficientes de difusão apresentaram magnitudes entre $3,93 \times 10^{-10}$ e $9,19 \times 10^{-10} \mathrm{~m}^{2} \mathrm{~s}^{-1}$, para o intervalo de temperatura de 30 a $70{ }^{\circ} \mathrm{C}$. Como já descrito por vários pesquisadores (ALMEIDA et al., 2009; CORRÊA et al., 2006; RESENDE, 2006; GONELI et al., 2007; RESENDE et al., 2009b), o coeficiente de difusão aumenta com a elevação da temperatura de secagem. 
Segundo GONELI et al. (2009), com o aumento da temperatura, a viscosidade da água diminui e, sendo a viscosidade uma medida de resistência do fluido ao escoamento, variações dessa propriedade implicam alterações na difusão da água nos capilares do grão de modo a favorecer a movimentação desse fluido no produto. No entanto, para o presente trabalho, o coeficiente de difusão foi ligeiramente maior na temperatura de $40{ }^{\circ} \mathrm{C}$ em relação à de $50{ }^{\circ} \mathrm{C}$, com valores de $5,14 \times 10^{-10}$ e $4,87 \times 10^{-10} \mathrm{~m}^{2} \mathrm{~s}^{-1}$, respectivamente. Contudo, esses valores não apresentaram diferença expressiva.

RESENDE et al. (2005) obtiveram valores de $6,32 \times 10^{-11}$ a $22,78 \times 10^{-11} \mathrm{~m}^{2} \cdot \mathrm{s}^{-1}$ para o arroz em casca, durante a secagem na faixa de temperatura entre $25^{\circ} \mathrm{C}$ e $55^{\circ} \mathrm{C}$. Desta forma, pode-se inferir que a água foi removida mais rapidamente nas sementes de pinhão-manso comparativamente aos grãos de arroz, devido a semente de pinhão ser uma oleaginosa e apresentar maior hidrofobicidade em relação ao grão de arroz, que é amiláceo.

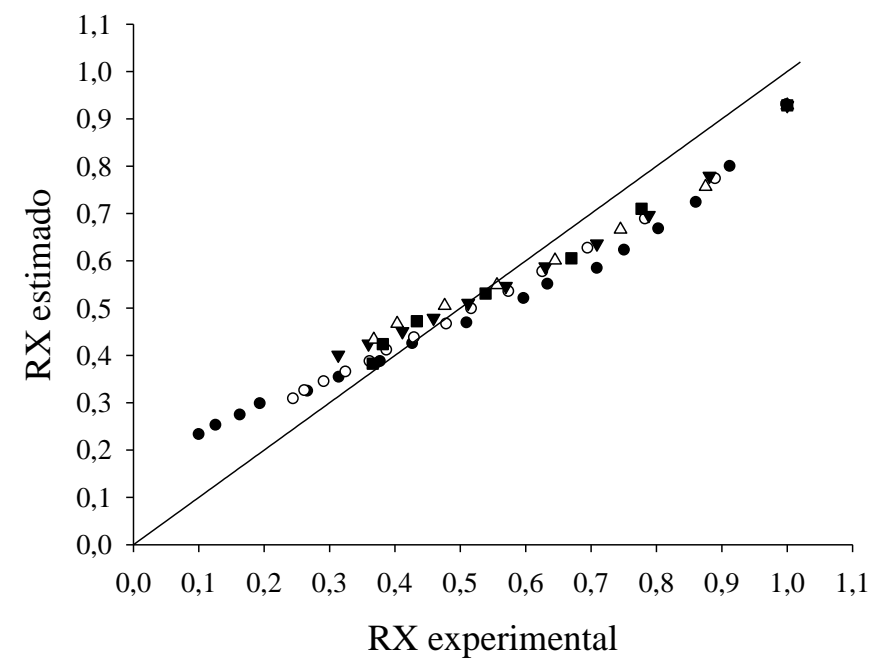

FIGURA 3. Valores experimentais e estimados de razão de teor de água pela estimativa dos parâmetros da equação de Midilli para a secagem do nabo-forrageiro. Experimental values and estimated ratio of water content by estimating the parameters of the equation for the radish drying.

Na Figura 3, estão representados os dados experimentais e calculados de razão de teor de água (RX) empregando-se a solução analítica do modelo da difusão líquida com os parâmetros estimados por meio da eq.(15). Verifica-se que há uma discrepância entre os valores experimentais e estimados pelo modelo, refletindo nos valores do coeficiente de determinação entre 88,1 e 94,7\%, erro médio relativo para as condições de $50 ; 60$ e $70{ }^{\circ} \mathrm{C}$ acima de $10 \%$ e erro médio estimado relativamente alto para a avaliação em estudo (Tabela 5). No entanto, estes parâmetros não comprometeram a obtenção do coeficiente de difusão efetivo por meio da solução analítica do modelo da difusão líquida.

TABELA 5. Coeficientes de determinação $\left(\mathrm{R}^{2}\right)$, erros médio relativo $(\mathrm{P})$ e estimado (SE) para solução analítica do modelo da difusão, durante a secagem do pinhão-manso em cinco condições de ar. Coefficients of determination (R2), the average relative error (P) and estimated (SE) for analytical solution of the diffusion model for jatropha drying in five air conditions.

\begin{tabular}{cccc}
\hline Temperatura $\left({ }^{\circ} \mathrm{C}\right)$ & $\mathrm{SE}($ decimal $)$ & $\mathrm{P}(\%)$ & $\mathrm{R}^{2}(\%)$ \\
\hline 30 & 0,1013812 & 29,73 & 88,1501 \\
40 & 0,0604623 & 10,45 & 93,3149 \\
50 & 0,0674582 & 10,15 & 90,7354 \\
60 & 0,0723294 & 9,90 & 89,8174 \\
70 & 0,0539160 & 7,35 & 94,7165 \\
\hline
\end{tabular}


A dependência do coeficiente de difusão com relação à temperatura do ar de secagem foi satisfatoriamente representada pela expressão de Ahrrenius, conforme ilustrado na Figura 4. Observa-se que os valores de Ln (D), em função do inverso da temperatura absoluta $\left(1 / \mathrm{T}_{\mathrm{a}}\right)$, apresentam comportamentos diferentes para os intervalos de temperatura de 30 a $50{ }^{\circ} \mathrm{C}$ e 50 a $70{ }^{\circ} \mathrm{C}$, em função do coeficiente de difusão efetivo para a temperatura de $40{ }^{\circ} \mathrm{C}$ ter sido sensivelmente superior ao da temperatura de $50{ }^{\circ} \mathrm{C}$. Assim, pode-se inferir que houve maior interferência das condições externas de secagem na temperatura de $40{ }^{\circ} \mathrm{C}$, como já descrito pela magnitude da constante de secagem (k).

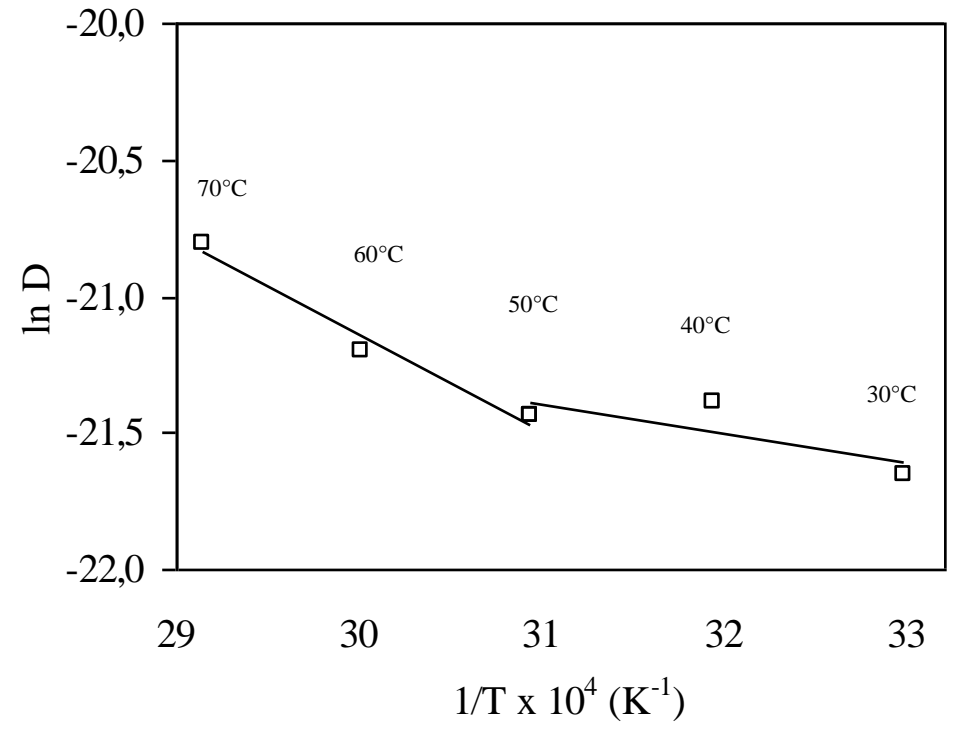

FIGURA 4. Representação de Arrhenius para o coeficiente de difusão efetivo, em função da temperatura do ar de secagem, obtido durante a secagem do pinhão-manso. Arrhenius representation for the effective diffusion coefficient as a function of drying air temperature obtained during the Jatropha drying.

A eq.(21) apresenta os coeficientes ajustados da expressão de Ahrrenius para o coeficiente de difusão efetivo durante a secagem do pinhão-manso.

$$
\mathrm{D}=2,3 \cdot 10^{-7} \cdot \operatorname{Exp}\left[\frac{-15,781}{\mathrm{R} \cdot \mathrm{Ta}}\right]
$$

Durante a determinação da energia de ativação, a interferência das condições externas de secagem na temperatura de $40{ }^{\circ} \mathrm{C}$ ocasionou um coeficiente de determinação no valor de $85,47 \%$. Termodinamicamente, a energia de ativação é definida como a facilidade com que as moléculas de água superam a barreira de energia durante e migração no interior do produto (CORRÊA et al., 2006). Sendo assim, quanto menor a energia de ativação maior será a velocidade com que a água será removida dos grãos. Conforme verificado na eq.(21), a energia de ativação para a difusão líquida do pinhão-manso foi de $15,781 \mathrm{~kJ} \mathrm{~mol}^{-1}$, sendo menor que a obtida por CORREA et al. (2006) trabalhando com grãos de feijão do grupo vermelho, com valor de $40,08 \mathrm{~kJ} \mathrm{~mol}^{-1}$, e similar à de mamona obtida por GONELI (2008), em que a energia de ativação apresentou o valor de $15,25 \mathrm{~kJ} \mathrm{~mol}^{-1}$.

A mamona é uma planta pertencente à mesma família do pinhão-manso (Euphorbiaceae); aliado a isso, as sementes de mamona apresentam propriedades similares às sementes da planta em estudo, como um tegumento lenhoso (rijo e duro) e, segundo NUNES et al. (2009), o endosperma desses grãos é de coloração branca tenra e rica em óleo. Isso explica a semelhança nos valores da energia de ativação entre os produtos. 


\section{CONCLUSÕES}

Nas condições em que foi desenvolvido o presente trabalho, conclui-se que, dentre os modelos analisados, Page e Henderson e Pabis Modificado apresentaram os melhores ajustes aos dados experimentais, sendo o modelo de Page selecionado para a descrição das curvas de secagem do pinhão-manso devido a sua simplicidade.

O aumento da temperatura de secagem promove maior difusividade de água nas sementes, e a energia de ativação para a difusão líquida do pinhão-manso foi de $15,781 \mathrm{~kJ} \mathrm{~mol}^{-1}$.

\section{REFERÊNCIAS}

ABALONE, R.; GASTÓN, A.; CASSINERA, A.; LARA, M.A. Thin layer drying of amaranth seeds. Biosystems Engineering, London, v.93, n.2, p.179-188, 2006.

AFONSO JÚNIOR, P.C.; CORRÊA, P.C. Comparação de modelos matemáticos para a descrição da cinética de secagem em camada fina de sementes de feijão. Revista Brasileira de Engenharia Agrícola e Ambiental, Campina Grande, v.3, n.3, p.349-353, 1999.

ALMEIDA, D.P.; RESENDE, O.; COSTA, L.M.; MENDES, U.C.; SALES, J.F. Cinética de secagem do feijão adzuki (Vigna angularis). Global Science and Technology, Rio Verde, v.2, n.1, p.72-83, 2009.

ANDRADE, E.T.; CORRÃ ŠA, P.C.; TEIXEIRA, L.P.; PEREIRA, R.G.; CALOMENI, J.F. Cinética de secagem e qualidade de sementes de feijão. Engevista, Niterói, v.8, n.2, p.83-95, 2006.

ARABHOSSEINIA, A.; HUISMANB, W.; VAN BOXTEL, A.; MÜLLER, J. Modeling of thin layer drying of tarragon (Artemisia dracunculus L.). Industrial Crops and Products, New Forests, v.29, n.1, p.53-59, 2009.

BABALIS, S.J.; BELESSIOTIS, V.G. Influence of the drying conditions on the drying constants and moisture diffusivity during the thin-layer drying of figs. Journal of Food Engineering, Essex, v.65, n.3, p.449-458, 2004.

BERBERT, P.A.; QUEIROZ, D.M.; SILVA, J.S.; PINHEIRO FILHO, J.B. Simulation of coffee drying in a fixed bed with periodic airflow reversal. Journal of Agricultural Engineering Research, v.60, n.3, p.167-173, 1995.

BRASIL. MINISTÉRIO DA AGRICULTURA E REFORMA AGRÁRIA. Secretaria Nacional de Defesa Agropecuária. Regras para análise de sementes. Brasília, 2009. 395 p.

BROOKER, D.B.; BAKKER-ARKEMA, F.W.; HALL, C.W. Drying and storage of grains and oilseeds. Westport: The AVI Publishing Company, 1992. 450 p.

CORRÊA, P.C.; RESENDE, O.; GONELI, A.L.D.; BOTELHO, F.M.; NOGUEIRA, B.L. Determinação do coeficiente de difusão liquida dos grãos de feijão. Revista Brasileira de Produtos Agroindustriais, Campina Grande, v.8, n.2, p.117-126, 2006.

CORRÊA, P.C.; RESENDE, O.; MARTINAZZO, A.P.; GONELI, A.L.D.; BOTELHO, F.M. Modelagem matemática para a descrição do processo de secagem do feijão (Phaseolus vulgaris L.) em camadas delgadas. Engenharia Agrícola, Jaboticabal, v.27, n.2, p.501-507, 2007.

DOYMAZ, I. Air-drying characteristics of tomatoes. Journal of Food Engineering, Essex, v.78, n.4, p.1.291-1.297, 2007.

GINER, S.A.; MASCHERONI, R.H. Diffusive drying kinetics in wheat. Part 2: applying the simplified analytical solution to experimental data. Postharvest Technology, Amsterdam, v.81, n.1, p.85-97, 2002. 
GONELI, A.L.D. Variação das propriedades físico-mecânicas e da qualidade de mamona (Ricinus communis L.) durante a secagem e o armazenamento. 2008. $186 \mathrm{f}$. Tese (Doutorado em Engenharia Agrícola) - Universidade Federal de Viçosa, Viçosa, 2008.

GONELI, A.L.D.; CORRÊA, P.C.; AFONSO JÚNIOR, P.C.; OLIVEIRA, G.H.H. Cinética de secagem dos grãos de café descascados em camada delgada. Revista Brasileira de Armazenamento, Viçosa-MG, n.11, p.64-73, 2009. Especial Café.

GONELI, A.L.D.; CORRÊA, P.C.; MAGALHÃES, F.E.A.; BAPTESTINI, F.M. Contração volumétrica e forma dos frutos de mamona durante a secagem. Acta Scientiarum Agronomy, Maringá, v.33, n.1, p.1-8, 2011.

GONELI, A.L.D.; CORRÊA, P.C.; RESENDE, O.; REIS NETO, S.A. Estudo da difusão de umidade em grãos de trigo durante a secagem. Ciência e Tecnologia de Alimentos, Campinas, v.27, n.1, p.135-140, 2007.

HENDERSON, S.M. Progress in developing the thin layer drying equation. Transactions of the $A S A E$, St. Joseph, v.17, p.1.167-1.168, 1974.

HENDERSON, S.M.; PABIS, S. Grain drying theory. Temperature effect on drying coefficient. Journal of Agricultural Engineering Research, London, n.6, p.169-174, 1961.

KARATHANOS, V.T. Determination of water content of dried fruits by drying kinetics. Journal of Food Engineering, London, v.39, p.337-344, 1999.

LEWIS, W.K. The drying of solid materials. Journal Industrial Engineering, London, n.5, p.427433, 1921.

MADAMBA, P.S.; DRISCOLL, R.H.; BUCKLE, K.A. Thin-layer drying characteristics of garlic slices. Journal of Food Engineering, London, v.29. n.1, p.75-97, 1996.

MIDILLI, A.; KUCUK, H.; YAPAR, Z.A. A new model for single-layer drying. Drying Technology, New York, v.20, n.7, p.1.503-1.513, 2002.

MISRA, M.K.; BROOKER, D.B. Thin layer drying and rewetting equations for shelled yellow corn. Transaction of the ASAE, St. Joseph, v.12, n.8, p.1.254-1.260, 1980.

MOHAPATRA, D.; RAO, P.S. A thin layer drying model of parboiled wheat. Journal of Food Engineering, Essex, v.66, n.4, p.513-518, 2005.

MOHSENIN, N.N. Physical properties of plant and animal materials. New York: Gordon and Breach Publishers, 1986. 841 p.

NUNES, C.F.; SANTOS, D.N.; PASQUAL, M.; VALENTE, T.C.T. Morfologia externa de frutos, sementes e plântulas de pinhão-manso. Pesquisa Agropecuária Brasileira, Brasília, v.44, n.2, p.207-210, 2009.

OLIVEIRA, R.A.; OLIVEIRA, W.P.; PARK, K.J. Determinação da difusividade efetiva de raiz de chicória. Engenharia Agrícola, Jaboticabal, v.26, n.1, p.181-189, 2006.

PAGE, G.E. Factors influencing the maximum rates of air drying shelled corn in thin layers. 1949. Thesis (M.Sc.) - Purdue University, West Lafayette, 1949.

RESENDE, O. Variação das propriedades físicas e mecânicas e da qualidade do feijão (Phaseolus vulgaris L.) durante a secagem e o armazenamento. 2006. $180 \mathrm{f}$. Tese (Doutorado em Engenharia Agrícola) - Universidade Federal de Viçosa, Viçosa, 2006.

RESENDE, O.; ARCANJO, R.V.; SIQUEIRA, V.C.; RODRIGUES, S. Modelagem matemática para a secagem de clones de café (Coffea canephora Pierre) em terreiro de concreto. Acta Scientiarum Agronomy, Maringá, v.31, n.2, p.189-196, 2009. 
RESENDE, O.; CORRÊA, P.C.; GONELI, A.L.D.; BOTELHO, F.M.; RODRIGUES, S. Modelagem matemática do processo de secagem de duas variedades de feijão (Phaseolus vulgaris L.). Revista Brasileira de Produtos Agroindustriais, Campina Grande, v.10, n.1, p.17-26, 2008.

RESENDE, O.; CORRÊA, P.C.; GONELI, A.L.D.; MARTINAZZO, A.P.; RIBEIRO, R.M. Contração volumétrica na difusão líquida durante o processo de secagem do arroz em casca. Revista Brasileira de Armazenamento, Viçosa-MG, v.30, n.2, p.163-171, 2005.

RESENDE, O.; RODRIGUES, E.; MENDES, U.C.; ALMEIDA, D.P. Hidratação e difusividade efetiva do feijão adzuki (Vigna angularis). Revista Brasileira de Armazenamento, Viçosa-MG, v.34, n.2, p.122-128, 2009b.

SHARAF-ELDEEN, Y. I.; BLAISDELL, J. L.; HAMDY, M. Y. A model for ear corn drying. Transactions of the ASAE, St. Joseph, v.23, p.1.261-1.265, 1980.

TEIXEIRA, L.C. Potencialidades de oleaginosas para produção de biodiesel. Informe Agropecuário, Belo Horizonte, v.26, n.229, 2005.

THOMPSON, T.L.; PEART, R.M.; FOSTER, G.H. Mathematical simulation of corn drying: A new model. Transactions of ASAE, St. Joseph, v.11, n.4, p.582-586, 1968.

VERMA, L.R.; BUCKLIN, R.A.; ENDAN, J.B.; WRATTEN, F.T. Effects of drying air parameters on rice drying models. Transactions of the ASAE, St. Joseph, v.28, p.296-301, 1985.

WANG, C.Y.; SINGH, R.P. Use of variable equilibrium moisture content in modeling rice drying. Transaction of ASAE, St. Joseph, v.11, n.A, 1978.

WANG, Z.; SUN, J.; CHEN, F.; LIAO, X.; HU, X. Mathematical modelling on thin layer microwave drying of apple pomace with and without hot air pre-drying. Journal of Food Engineering, London, v.80, n.2, p.536-544, 2007.

YAGCIOGLU, A.; DEGIRMENCIOGLU, A.; CAGATAY, F. Drying characteristics of laurel leaves under different conditions. In: INTERNATIONAL CONGRESS ON AGRICULTURAL MECHANIZATION AND ENERGY, 7., 1999, Adana: Faculty of Agriculture, Cukurova University, 1999. p.565-569. Proceedings... 